\title{
Ultrasonic Nonlinearity Evaluation of the Cracked Interface
}

\author{
Yanjun Qiu, ${ }^{1}$ Yilin Zhang, ${ }^{1}$ Yewang $\mathrm{Su},{ }^{2}$ Peng Cao, ${ }^{3}$ and Youxuan Zhao ${ }^{4}$ \\ ${ }^{1}$ School of Civil Engineering, Southwest Jiaotong University, Chengdu 610031, China \\ ${ }^{2}$ State Key Laboratory of Nonlinear Mechanics, Institute of Mechanics, Chinese Academy of Sciences, Beijing 100190, China \\ ${ }^{3}$ Department of Hydraulic Engineering, Tsinghua University, Beijing 100084, China \\ ${ }^{4}$ College of Aerospace Engineering, Chongqing University, Chongqing 400044, China
}

Correspondence should be addressed to Youxuan Zhao; youxuan.zhao@gmail.com

Received 14 December 2015; Revised 5 May 2016; Accepted 15 May 2016

Academic Editor: Roman Lewandowski

Copyright (C) 2016 Yanjun Qiu et al. This is an open access article distributed under the Creative Commons Attribution License, which permits unrestricted use, distribution, and reproduction in any medium, provided the original work is properly cited.

\begin{abstract}
This paper derives a novel analytical solution for acoustic nonlinearity evaluation of the cracked interface. When microcracks exist at the interface, the tensile and compressive effective moduli of the cracked interface are considered to be different. It is clearly shown that the tension and compression elastic asymmetry can result in acoustic nonlinearity. In addition, numerical simulations using the finite element method are conducted to validate the theoretical solution. It is shown that numerical results agree well with the analytical solution. Finally, two factors affecting acoustic nonlinearity are studied based on the analytical solution. One is the tension and compression elastic asymmetry and another is the frequency of incident wave. Different from acoustic nonlinearity parameter of the general second harmonics, it is found that acoustic nonlinearity parameter is a function of two factors.
\end{abstract}

\section{Introduction}

Adhesion bonding technology for fiber reinforced composite has been widely applied in aerospace industry. Generally, the cohesive interface is very thin but can provide the powerful strength and resiliency. However, the capability of the cohesive interface could be greatly affected by the imperfect condition, such as debond, fatigue damage, and microcracks. Thus, there is a great need for development of nondestructive testing methods to detect the imperfect condition.

Nonlinear ultrasonic methods have the powerful ability to characterize the material nonlinearity change caused by plasticity [1], imperfect interface [2-4], microcracks $[5$, $6]$, and fatigue damage $[7,8]$. When a time-harmonic longitudinal wave propagates through a cracked solid or interface, it will cause the tension and compression elastic asymmetry; then the waveform will be distorted and higherorder harmonic waves are generated [9-11]. And considerable experimental evidence $[12,13]$ has shown that ultrasonic waves do interact with microcracks in a nonlinear fashion, but those researches may fail when the crack size is only tens of microns, which is one of the initial factors leading to interface degradation.
For the researches on acoustic nonlinearity induced by microcracks [14-16], majority are concentrated either on the scattering of elastic waves by a single crack or an array of cracks [17-20] or on the propagation of elastic waves in a cracked medium [21-23]. The first study on such contact-induced acoustic nonlinearity is probably the paper by Richardson [24] who considered the contact interface between two semi-infinite half-spaces. He has analyzed one-dimensional nonlinear wave propagation in a system composed of an unbounded planer interface separating two semi-infinite linear elastic media. The nonlinearity is caused by the opening and closing of the interface. However, in Richardson's analysis, the interface stiffness varying continuously is not accounted for. Improving Richardson's theory, Biwa et al. [25] have analyzed a nonlinear interface stiffness model, where the stiffness property of the contact interface is described as a function of the nominal contact pressure. Furthermore, by assuming the interface of the adhesive as a nonlinear spring, Achenbach and Parikh [26] have investigated theoretically to obtain information on the adhesive bond strength from ultrasonic test results, and it is shown that the nonlinear adhesive bond behavior could cause the generation of higher harmonics. 
In this paper, based on the researches of Richardson and Biwa, one theoretical solution for one-dimensional nonlinear wave propagation in a system composed of a multicrackincluded interface separating two semi-infinite elastic media is derived, from which the acoustic nonlinearity caused by the tension and compression elastic asymmetry is clearly shown. To validate the analytical solution, numerical results from the FEM simulations are presented. Comparison between the analytical predictions and the FEM simulation results shows good agreement. Finally, we also study two factors affecting acoustic nonlinearity. One is the tension and compression elastic asymmetry of the cracked interface and another is the frequency of incident wave.

\section{Solution for Harmonic Wave Incidence}

We consider a system composed of a cracked interface separating two semi-infinite elastic media. In the presence of microcracks, the interface will respond to the tensile and compressive loadings differently, which is the tension and compression elastic asymmetry. According to [11], the elastic constants of the cracked medium can be considered to be dependent with crack density, the friction of the crack faces, and frequency of incident wave:

$$
\begin{aligned}
& \frac{\bar{E}_{t}^{\prime}}{E^{\prime}}=\frac{1}{1+\pi d h_{1}^{t}}, \\
& \bar{\nu}_{t}^{\prime}=\frac{\nu^{\prime}-\pi d h_{2}^{t}}{1+\pi d h_{1}^{t}}, \\
& \frac{\bar{E}_{c}^{\prime}}{E^{\prime}}=\frac{1}{1+\pi d h_{1}^{c}}, \\
& \bar{\nu}_{c}^{\prime}=\frac{v^{\prime}+\pi c h_{1}^{c}}{1+\pi d h_{1}^{c}},
\end{aligned}
$$

where $E^{\prime}$ and $\nu^{\prime}$ are Young's modulus and Poisson's ratio of the uncracked solid, respectively. And $\bar{E}_{\alpha}$ and $\bar{\nu}_{\alpha}$ are, respectively, the corresponding effective Young's modulus and Poisson's ratio of the cracked solid under tension $(\alpha=t)$ and under compression $(\alpha=c)$. Meanwhile, $E^{\prime}=E$ and $\nu^{\prime}=\nu$ for plane stress, and $E^{\prime}=E /\left(1-v^{2}\right)$ and $\nu^{\prime}=v /(1-v)$ for plane strain. The crack density $d$ is defined as $d=N a^{2} / A$, where $a$ is the average half-length of the cracks; $A$ is the area of the solid containing $N$ randomly distributed and randomly oriented two-dimensional microcracks.

We have

$$
\begin{aligned}
h_{1}^{t} & =1-A \eta^{2} \ln \eta+\left[\frac{\kappa^{2}-1+\kappa^{4}(1-2 \log \kappa)}{16 \kappa^{2}\left(\kappa^{2}-1\right)}\right. \\
& +A(\log 4+\log \kappa-\gamma)] \eta^{2}+\frac{i \pi A}{2} \eta^{2},
\end{aligned}
$$

$$
\begin{aligned}
h_{2}^{t} & =-B \eta^{2} \ln \eta+\left[\frac{1+2 \gamma-2 \gamma \kappa^{2}-2 \log 2}{32 \kappa^{2}}\right. \\
& +B(\log 2-\log \kappa)] \eta^{2}+\frac{i \pi B}{2} \eta^{2} \\
h_{1}^{c} & =\frac{F}{4}-\frac{F\left(\kappa^{4}+1\right)}{32 \kappa^{2}\left(\kappa^{2}-1\right)} \eta^{2} \ln \eta \\
& +\frac{F\left[\kappa^{4}+2\left(1+\kappa^{4}\right)(\log 4-\gamma)+2 \log \kappa\right]}{64 \kappa^{2}\left(\kappa^{2}-1\right)} \eta^{2} \\
& -\frac{1}{32 \pi \kappa^{2}}\left[\tan ^{-1} \frac{1}{\mu}-\frac{\mu\left(3 \mu^{2}+1\right)}{3\left(\mu^{2}+1\right)^{2}}\right] \eta^{2} \\
& +\frac{i \pi F\left(\kappa^{4}+1\right)}{64 \kappa^{2}\left(\kappa^{2}-1\right)} \eta^{2} .
\end{aligned}
$$

In the above, $\gamma$ is the Euler-Mascheroni constant, $\eta=$ $k_{T} a=\omega a / c_{T}$ is a dimensionless wavenumber, $\kappa=c_{L} / c_{T}=$ $\sqrt{2(1-v) /(1-2 \nu)}$ is a dimensionless parameter, $\mu$ is the coefficient of friction, and

$$
\begin{aligned}
& A=\frac{5-6 \kappa^{2}+5 \kappa^{4}}{16 \kappa^{2}\left(\kappa^{2}-1\right)} \\
& B=\frac{\kappa^{2}-1}{16 \kappa^{2}} \\
& F=\frac{2}{\pi}\left(\tan ^{-1} \frac{1}{\mu}-\frac{\mu}{\mu^{2}+1}\right) .
\end{aligned}
$$

Therefore, in this paper, the conclusion of [11] will be adopted, and the interface can be regarded as an equivalent medium consisting of the random distribution cracks. Actually this assumption has been proven in [25] for investigating the void inclusion medium subjected to the ultrasonic wave loading conditions.

2.1. The General Solution. Firstly, we assume that the elasticity of the cracked interface is a function of time, and the stress in the interface is uniform. In Figure 1 we illustrate a one-dimensional system schematically to consider elastic longitudinal wave propagation along $x$-axis, where $f(x-c t)$ and $g(x-c t)$ represent the incident and transmitted wave functions, respectively. The blue area represents the cracked interface.

The two semi-infinite elastic solids are located in the regions $x<0^{-}$and $x>0^{+}$, respectively. The equation of responses of the interface and the stress-strain relation are defined as follows:

$$
\begin{aligned}
\rho \frac{\partial^{2} u(x, t)}{\partial^{2} t} & =\frac{\partial \sigma(x, t)}{\partial x}, \\
\sigma(x, t) & =E_{s}(t) \frac{\partial u(x, t)}{\partial x},
\end{aligned}
$$




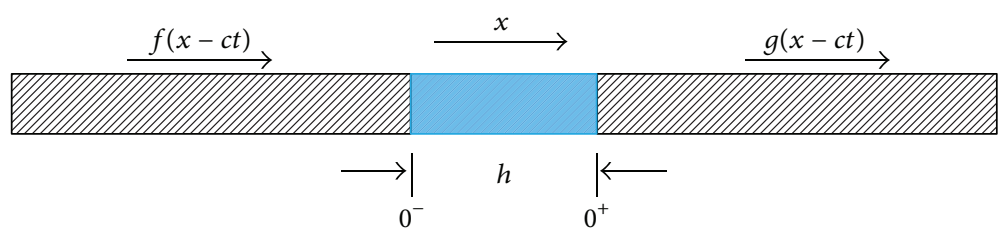

FIGURE 1: One-dimensional simplistic model.

where $\rho$ is mass density, $E_{s}(t)$ is the longitudinal stiffness of the cracked interface, $u(x, t)$ is the displacement in the $x$ direction of an element at time $t$ from its position $x$, and $\sigma(x, t)$ is the stress. The boundary conditions in locations $0^{-}$ and $0^{+}$are as follows:

$$
\begin{aligned}
\sigma(t) & =\sigma\left(0^{-}, t\right)=\sigma\left(0^{+}, t\right), \\
u\left(0^{+}, t\right)-u\left(0^{-}, t\right) & =\sigma(t) \cdot \frac{h}{E_{s}},
\end{aligned}
$$

where $h$ is the thickness of the cracked interface.

According to $[24,25]$, the governing equation of (4) can be solved based on travelling wave method. Therefore, this paper will employ travelling wave method to obtain the general displacement solution when longitudinal wave propagates through the interface. Then considering the cracked interface as the tension and compression elastic asymmetry, this paper will achieve the corresponding special displacement solution.

According to the travelling wave method, as a solution to (4), the following forms are considered [24, 25]:

$$
\begin{aligned}
u(x, t) & =f(x-c t)-\frac{1}{2} V\left(t+\frac{x}{c}\right), \quad x<0^{-}, \\
u(x, t) & =f(x-c t)+\frac{1}{2} V\left(t-\frac{x}{c}\right), \quad x \geq 0^{+}, \\
V(t) & =u\left(0^{+}, t\right)-u\left(0^{-}, t\right),
\end{aligned}
$$

where $c=\sqrt{E / \rho}$ is the wave velocity, $E$ is the longitudinal stiffness of the two semi-infinite elastic solids, $f(x-c t)$ represents the incident waves, the term $-(1 / 2) V(t+x / c)$ represents reflected waves, and (8) represents the transmitted waves. Equation (9) represents the equivalent displacement of the cracked interface.

According to (6) and (9), we can obtain

$$
2 V(t)=\frac{h E}{E_{s}}\left[\left.2 \frac{\partial f(x-c t)}{\partial(x-c t)}\right|_{x=0}-\frac{1}{c} \frac{d V(t)}{d t}\right] .
$$

The incident wave $f(x-c t)$ is now assumed in the following form:

$$
f(x-c t)=F_{0} \cos [k(x-c t)],
$$

where $k$ is the wave number and $F_{0}$ is the maximum amplitude.

Substitution of (11) in (10) yields

$$
\frac{d V(t)}{d t}+\xi V(t)=2 F_{0} \omega \sin (\omega t)
$$

where $\omega$ is the angular frequency and $\xi=\xi(t)=2 c E_{s}(t) / h E$.
Therefore, the general solution of (12) is

$$
\begin{aligned}
V(t)= & e^{-\int \xi(t) d t} \int 2 F_{0} \omega \sin (\omega t) e^{\int \xi(t) d t} d t \\
& +C_{1} e^{-\int \xi(t) d t} .
\end{aligned}
$$

When we know the function form of $\xi(t)$, we can obtain the special solution from (13).

2.2. The Special Solution. Assuming the different tensile and compression effective moduli of the cracked interface from [11], the function $E_{s}(t)$ can be defined by

$$
E_{s}(t)= \begin{cases}E_{s}^{t}, & \text { when } \sigma(t)>0 \\ E_{s}^{c}, & \text { when } \sigma(t)<0\end{cases}
$$

where $E_{s}^{t}$ and $E_{s}^{c}$ are the tensile and compression effective moduli, respectively. And the parameter $\gamma=\left(E_{s}^{t}-E_{s}^{c}\right) / E$ is introduced to represent the tension and compression asymmetry in the elastic moduli of the cracked interface.

According to (13) and (14), we can obtain

$$
\begin{aligned}
& V(t) \\
& \quad=2 F_{0} \sin \varphi\left[\sin \left(\omega t_{n}+\varphi\right) e^{-\xi\left(t-t_{n}\right)}-\sin (\omega t+\varphi)\right],
\end{aligned}
$$

where $\varphi=\sin ^{-1}\left(-\omega / \sqrt{\xi^{2}+\omega^{2}}\right)$.

Since $V\left(t_{n}\right)=0$ and the calculation could begin from $t_{0}=$ 0 without loss of generality, the time points $t_{n+1}$ to switch the property come from the following equation:

$$
\begin{aligned}
\sin \left(\omega t_{n}+\varphi_{t}\right) e^{-\xi_{t}\left(t_{n+1}-t_{n}\right)}-\sin \left(\omega t_{n+1}+\varphi_{t}\right) & =0 \\
n= & 0,2,4, \ldots \\
\sin \left(\omega t_{n}+\varphi_{c}\right) e^{-\xi_{c}\left(t_{n+1}-t_{n}\right)}-\sin \left(\omega t_{n+1}+\varphi_{c}\right) & =0 \\
n & =1,3,5, \ldots
\end{aligned}
$$


The displacement becomes

$$
V(t)= \begin{cases}2 F_{0} \sin \varphi_{t}\left[\sin \left(\omega t_{n}+\varphi_{t}\right) e^{-\xi_{t}\left(t-t_{n}\right)}-\sin \left(\omega t+\varphi_{t}\right)\right], & n=0,2,4, \ldots \\ 2 F_{0} \sin \varphi_{c}\left[\sin \left(\omega t_{n}+\varphi_{c}\right) e^{-\xi_{c}\left(t-t_{n}\right)}-\sin \left(\omega t+\varphi_{c}\right)\right], & n=1,3,5, \ldots\end{cases}
$$

where

$$
\begin{aligned}
& \varphi_{t}=\sin ^{-1}\left(\frac{-\omega}{\sqrt{\xi_{t}^{2}+\omega^{2}}}\right), \quad \xi_{t}=\frac{2 c E_{s}^{t}}{h E}, \\
& \varphi_{c}=\sin ^{-1}\left(\frac{-\omega}{\sqrt{\xi_{c}^{2}+\omega^{2}}}\right), \quad \xi_{c}=\frac{2 c E_{s}^{c}}{h E} .
\end{aligned}
$$

Substitution of (17) back to (7) and (8) gives the finial displacement field of the problem.

\section{Analytical and FEM Results}

3.1. Analytical Results. Numerical solutions using Matlab are performed to solve (16). For a demonstrative purpose, dashed line in Figure 2 shows one example of transmitted waveforms for an incident wave with the center frequency of $1 \mathrm{MHz}$, obtained by numerical solutions of (16) and (17), with the parameters $\rho=2700 \mathrm{~kg} / \mathrm{m}^{3}, E=7 \times 10^{10} \mathrm{~Pa}, E_{s}^{t}=7 \times 10^{9} \mathrm{~Pa}$, $E_{s}^{c}=7 \times 10^{10} \mathrm{~Pa}, F_{0}=1 \times 10^{-5} \mathrm{~m}$, and $h=1 \times 10^{-4} \mathrm{~m}$. The time-domain waveform with the dashed line is shown in Figure 2(a), and the locals in Figures 2(b1) and 2(b2) are to compare the difference of the displacement curve subjected to the first-half and second-half waveform in one-cycle loading conditions. It is seen that the first-half waveform in one cycle is distinctly different from the second-half waveform caused by the tension and compression elastic asymmetry. And Figure 2(c) shows the frequency spectrum using FFT method; it is clearly shown that the transmitted wave contains the term with angular frequency $2 \omega$, which is second harmonic generated by the cracked interface.

3.2. Comparison with FEM Results. The analytical solutions will be validated in this section by conducting numerical simulations of wave propagation through a cracked interface. The numerical simulations are performed using the finite element method (FEM) for the case of two-dimensional plane strain deformation. The commercial FEM software ABAQUS is used for this purpose.

To this end, a two-dimensional FEM model is constructed using the four-node plane strain (CPE4R) elements. To simulate plane waves, a rectangular strip of $0.09 \mathrm{~m} \times 0.003 \mathrm{~m}$ is used in our FEM model. The model consists of $\sim 300,000$ four-node plane strain (CPE4R) elements. The user defined constitutive law is performed to present the different tension and compression elasticity of the cracked interface. And the interface is located in the middle of the sample with the thickness of $1 \times 10^{-4} \mathrm{~m}$. Other regions are the isotropic elastic material of aluminum. A plane longitudinal wave is generated from one end of the sample. The amplitude longitudinal wave with the frequency of $1 \mathrm{MHz}$ is $1 \times 10^{-5} \mathrm{~m}$. The receiver is located at right end of the interface.

Shown in Figure 2(a) with the solid line is the FEM result of time-domain waveform of transmitted wave. The excellent comparison shows that the analytical solution results are identical to that of the FEM simulation. Furthermore, the two obvious amplitudes, being $9.37 \times 10^{-6} \mathrm{~m}$ and $7.76 \times 10^{-7} \mathrm{~m}$, respectively, exist with the frequencies of ultrasonic wave at $1 \mathrm{MHz}$ and $2 \mathrm{MHz}$ in Figure 2(c).

\section{Acoustic Nonlinearity Parameter}

The time-domain waveform curve can be obtained by the analytical solution derived in Section 2; then the frequency spectrum using FFT method can also be solved to obtain $A_{1}$ and $A_{2}$, which are the amplitudes of the fundamental and second harmonic, respectively. Through the analysis of data from the analytical solution, we can obtain several observations. First, $A_{2}$ is linearly related to $A_{1}$. This is significantly different from the second harmonic generated by quadratic nonlinearity in the solid, where $A_{2}$ is proportional to the square of $A_{1}$. The fundamental cause of this relationship is the tension and compression elastic asymmetry of the cracked interface. Second, $A_{2}$ is scaled by parameter $\gamma$. Therefore, according to [9], for acoustic nonlinearity parameter in cracked solids, the similar acoustic nonlinearity parameter of the cracked interface is introduced by

$$
\beta=\frac{3 \pi A_{2}}{A_{1} k h}
$$

For investigating the relation between the linearity parameter and the frequencies of the ultrasonic acoustic, a series of analytic solutions have been performed under different frequencies including $0.5 \mathrm{MHz}, 1 \mathrm{MHz}, 1.5 \mathrm{MHz}$, 2.0 $\mathrm{MHz}$, and $2.5 \mathrm{MHz}$. Figure 3 shows the acoustic nonlinearity parameter versus frequency. It is seen that although the acoustic nonlinearity parameter decreases linearly with increasing frequency, the decrease is not significant. In other words, the acoustic nonlinearity parameter has a rather weak dependence on frequency.

Figure 4 shows the acoustic nonlinearity parameter versus parameter $\gamma$. It is seen that the acoustic nonlinearity parameter increases linearly with increasing $\gamma$, which can represent the degree of damage for the cracked interface. In other words, the degree of damage for the cracked interface can be characterized by the acoustic nonlinearity parameter defined as (19). 


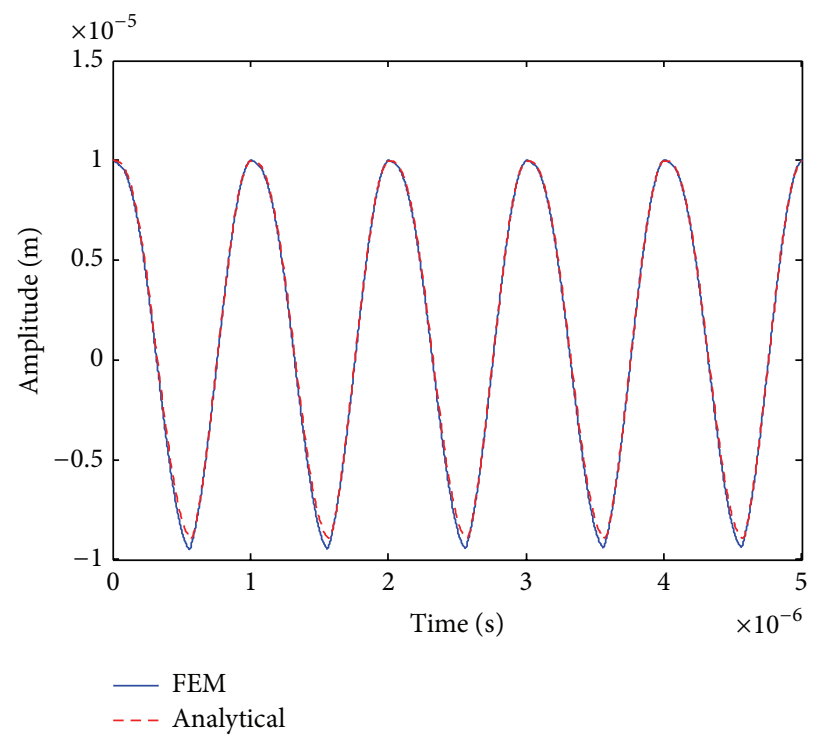

(a) Time-domain waveform

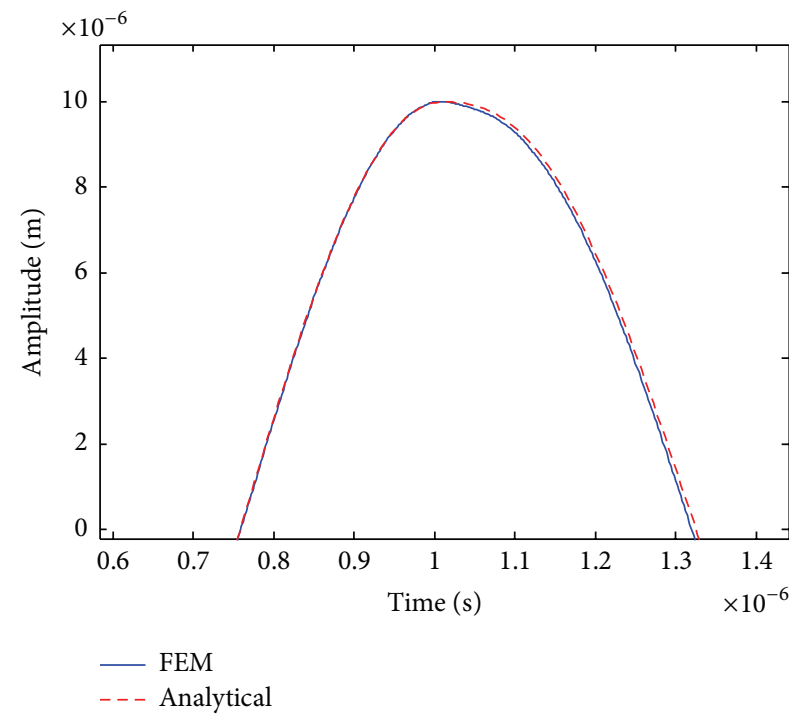

(b1) First-half waveform in one cycle

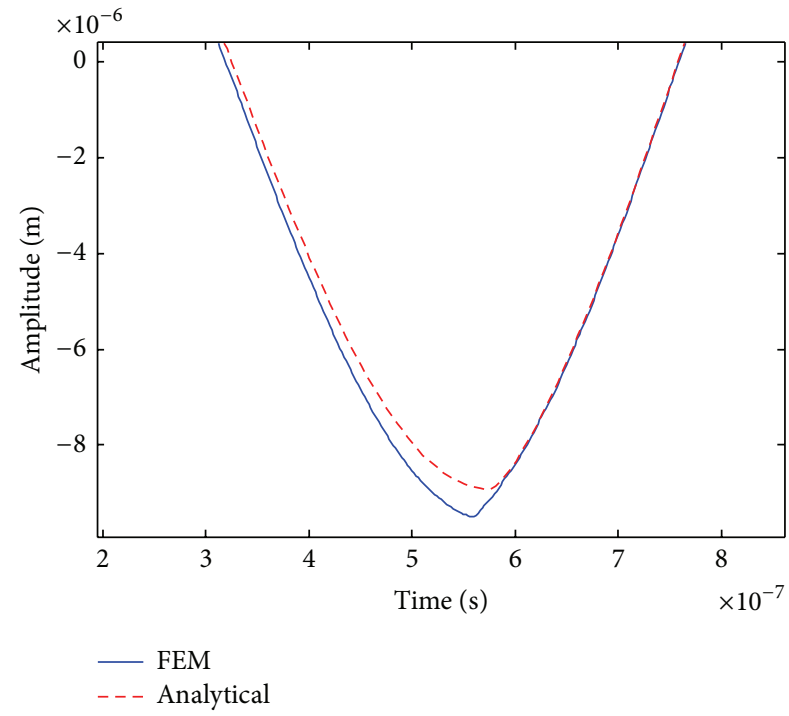

(b2) Second-half waveform in one cycle

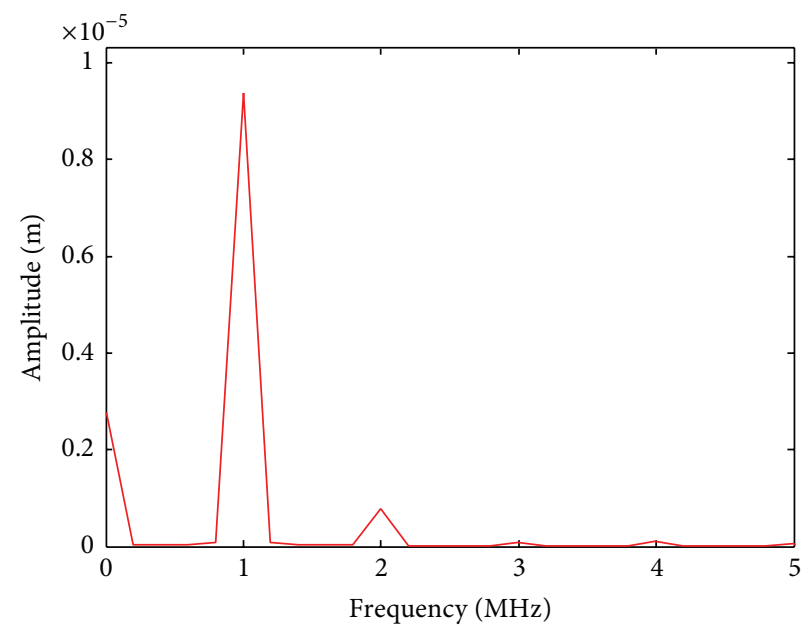

(c) Frequency spectrum using FFT method

FiguRE 2: Waveforms (a), (b1), and (b2) and frequency spectrum (c) of transmitted wave at location $x=0^{+}$. 


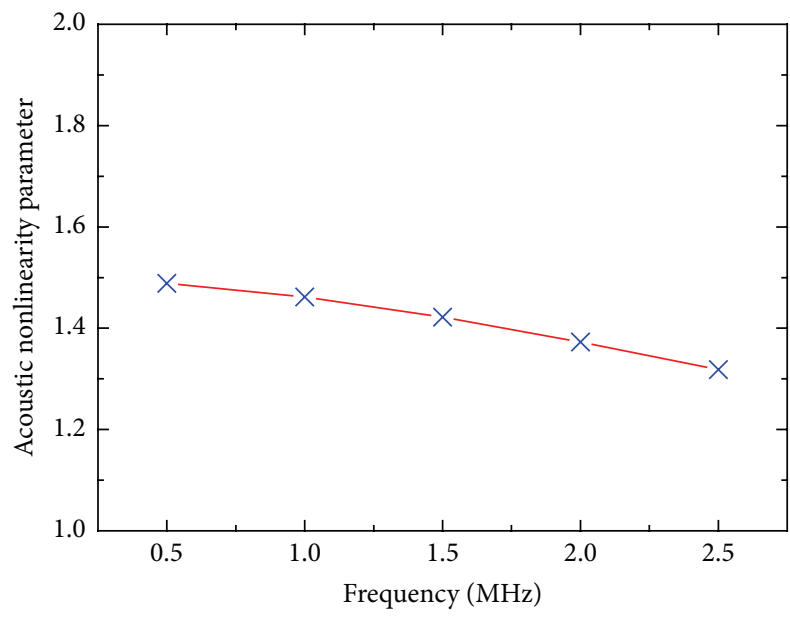

FIGURE 3: Acoustic nonlinearity parameter versus frequency.

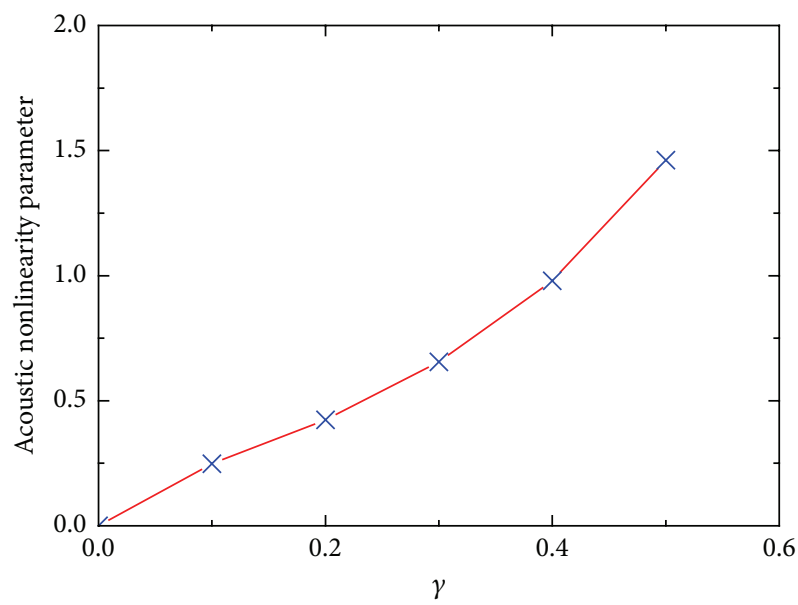

FIGURE 4: Acoustic nonlinearity parameter versus parameter $\gamma$.

\section{Conclusions}

As a time-harmonic longitudinal wave propagates in a system composed of a cracked interface separating two semi-infinite elastic media, a second harmonic wave may be generated by the cracked interface. Assuming the longitudinal stiffness of the interface as a function of time, this paper solves the problem and obtains the analytical general solution (any interface) and the special solution (the cracked interface). It is clearly shown that the tension and compression elastic asymmetry can result in acoustic nonlinearity. Furthermore, to verify the developed theoretical solution, we carry out detailed numerical simulation of wave propagation through a cracked interface by using the FEM. The results indicate that the micromechanics model predictions agree well with the FEM simulations. Finally, because the amplitude of the second harmonic is linearly related to the amplitude of the fundamental harmonic, we introduce the acoustic nonlinearity parameter induced by the cracked interface based on the analytical solution. It is shown that acoustic nonlinearity parameter is a function of two factors, which are the tension/compression elastic asymmetry and the frequency of incident wave, respectively. This study is beneficial to develop nonlinear ultrasonic quantitative NDE techniques for assessing microcrack-induced damage of interfaces.

In future, we will design and develop the reasonable experiments to validate the solution of this paper.

\section{Competing Interests}

The authors declare that there are no competing interests regarding the publication of this paper.

\section{Acknowledgments}

Youxuan Zhao acknowledges the support from the Fundamental Research Funds for the Central Universities (Project no. 0903005203383). Yewang Su acknowledges the support from Chinese Academy of Sciences via the "Hundred Talent program” and support from NSFC (no. 11572323).

\section{References}

[1] A. J. Croxford, P. D. Wilcox, B. W. Drinkwater, and P. B. Nagy, "The use of non-collinear mixing for nonlinear ultrasonic detection of plasticity and fatigue," The Journal of the Acoustical Society of America, vol. 126, no. 5, pp. EL117-EL122, 2009.

[2] J. Jiao, J. Sun, N. Li, G. Song, B. Wu, and C. He, "Micro-crack detection using a collinear wave mixing technique," NDT and $E$ International, vol. 62, pp. 122-129, 2014.

[3] S. Zhou and Y. Shui, "Nonlinear reflection of bulk acoustic waves at an interface," Journal of Applied Physics, vol. 72, no. 11, pp. 5070-5080, 1992.

[4] G. Shui, Y.-S. Wang, P. Huang, and J. Qu, "Nonlinear ultrasonic evaluation of the fatigue damage of adhesive joints," NDT and E International, vol. 70, pp. 9-15, 2015.

[5] V. V. Kazakov and A. M. Sutin, "Pulsed sounding of cracks with the use of the modulation of ultrasound by vibrations," Acoustical Physics, vol. 47, no. 3, pp. 308-312, 2001.

[6] D. T. Zeitvogel, K. H. Matlack, J.-Y. Kim, L. J. Jacobs, P. M. Singh, and J. Qu, "Characterization of stress corrosion cracking in carbon steel using nonlinear Rayleigh surface waves," NDT and E International, vol. 62, pp. 144-152, 2014.

[7] P. B. Nagy, "Fatigue damage assessment by nonlinear ultrasonic materials characterization," Ultrasonics, vol. 36, no. 1-5, pp. 375381, 1998.

[8] G. P. M. Fierro, F. Ciampa, D. Ginzburg, E. Onder, and M. Meo, "Nonlinear ultrasound modelling and validation of fatigue damage," Journal of Sound and Vibration, vol. 343, pp. 121-130, 2015.

[9] Y. Zhao, Y. Qiu, L. J. Jacobs, and J. Qu, "A micromechanics model for the acoustic nonlinearity parameter in solids with distributed microcracks," AIP Conference Proceedings, vol. 1706, Article ID 060001, 2016.

[10] Y. Zhao, Y. Qiu, L. J. Jacobs, and J. Qu, "Frequency-dependent tensile and compressive effective moduli of elastic solids with distributed penny-shaped microcracks," Acta Mechanica, vol. 227, no. 2, pp. 399-419, 2016.

[11] Y. Zhao, Y. Qiu, L. J. Jacobs, and J. Qu, "Frequency-dependent tensile and compressive effective moduli of elastic solids with 
randomly distributed two-dimensional microcracks," Journal of Applied Mechanics, vol. 82, no. 8, Article ID 081006, 2015.

[12] K. Jinno, A. Sugawara, Y. Ohara, and K. Yamanaka, "Analysis on nonlinear ultrasonic images of vertical closed cracks by damped double node model," Materials Transactions, vol. 55, no. 7, pp. 1017-1023, 2014.

[13] J. Rivière, M. C. Remillieux, Y. Ohara et al., "Dynamic acoustoelasticity in a fatigue-cracked sample," Journal of Nondestructive Evaluation, vol. 33, no. 2, pp. 216-225, 2014.

[14] G. Sih and J. Loeber, "Normal compression and radial shear waves scattering at a penny-shaped crack in an elastic solid," The Journal of the Acoustical Society of America, vol. 46, no. 3, pp. 711-721, 1969.

[15] A. K. Mal, "Interaction of elastic waves with a Griffith crack," International Journal of Engineering Science, vol. 8, no. 9, pp. 763-776, 1970.

[16] A. K. Mal, "Interaction of elastic waves with a penny-shaped crack," International Journal of Engineering Science, vol. 8, no. 5, pp. 381-388, 1970.

[17] J. D. Achenbach, Ray Methods for Waves in Elastic Solids: With Applications to Scattering by Cracks, Edited by A. K. Gautesen and H. McMaken, Pitman Advanced Publishing, Boston, Mass, USA, 1982.

[18] J. D. Achenbach and A. N. Norris, "Loss of specular reflection due to nonlinear crack-face interaction," Journal of Nondestructive Evaluation, vol. 3, no. 4, pp. 229-239, 1982.

[19] Y. C. Angel and J. D. Achenbach, "Reflection and transmission of elastic waves by a periodic array of cracks," Journal of Applied Mechanics, vol. 52, no. 1, pp. 33-41, 1985.

[20] Y. C. Angel and J. D. Achenbach, "Reflection and transmission of elastic waves by a periodic array of cracks: oblique incidence," Wave Motion, vol. 7, no. 4, pp. 375-397, 1985.

[21] V. E. Nazarov and A. M. Sutin, "Nonlinear elastic constants of solids with cracks," Journal of the Acoustical Society of America, vol. 102, no. 6, pp. 3349-3354, 1997.

[22] A. S. Eriksson, A. Boström, and S. K. Datta, "Ultrasonic wave propagation through a cracked solid," Wave Motion, vol. 22, no. 3, pp. 297-310, 1995.

[23] D. Gross and C. Zhang, "Wave propagation in damaged solids," International Journal of Solids and Structures, vol. 29, no. 14-15, pp. 1763-1779, 1992.

[24] J. M. Richardson, "Harmonic generation at an unbonded interface-I. Planar interface between semi-infinite elastic media," International Journal of Engineering Science, vol. 17, no. 1, pp. 73-85, 1979.

[25] S. Biwa, S. Nakajima, and N. Ohno, "On the acoustic nonlinearity of solid-solid contact with pressure-dependent interface stiffness," Journal of Applied Mechanics, vol. 71, no. 4, pp. 508515, 2004.

[26] J. D. Achenbach and O. K. Parikh, "Ultrasonic analysis of nonlinear response and strength of adhesive bonds," Journal of Adhesion Science and Technology, vol. 5, no. 8, pp. 601-618, 1991. 


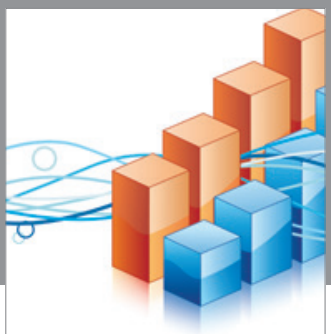

Advances in

Operations Research

vatem alat4

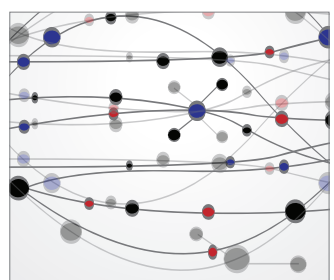

\section{The Scientific} World Journal
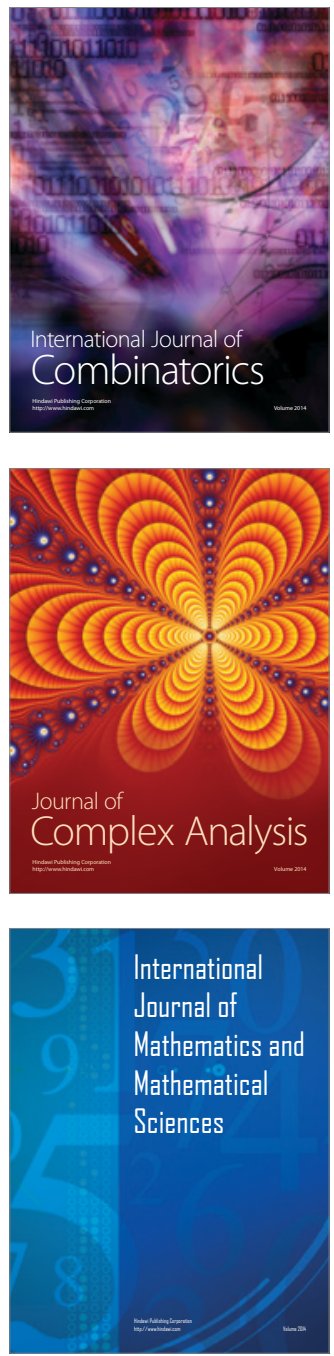
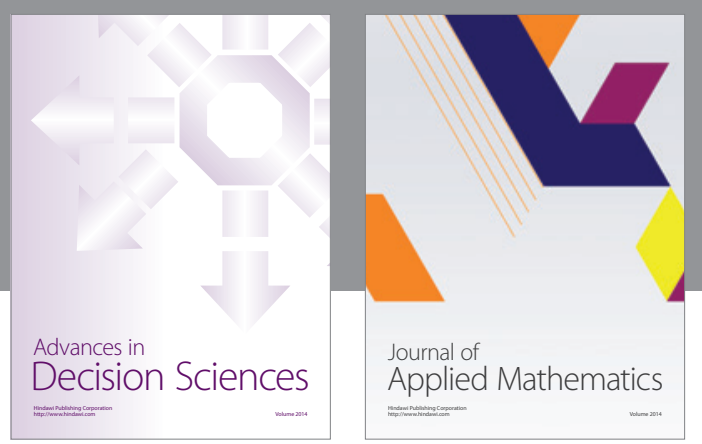

Algebra

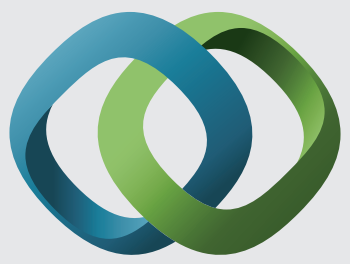

\section{Hindawi}

Submit your manuscripts at

http://www.hindawi.com
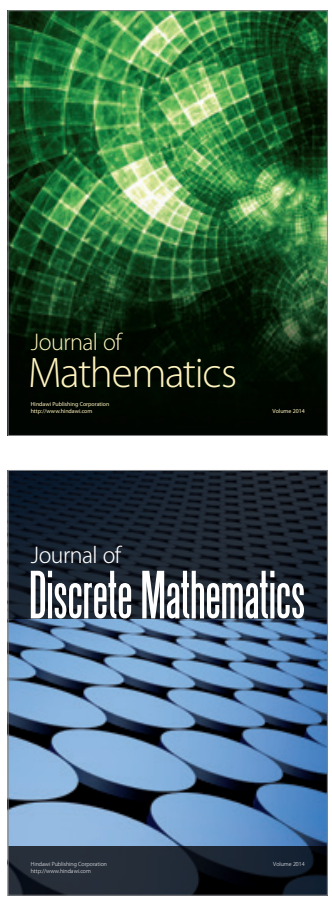

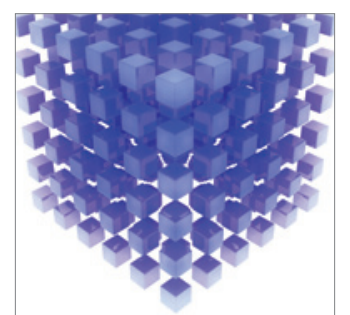

Mathematical Problems in Engineering
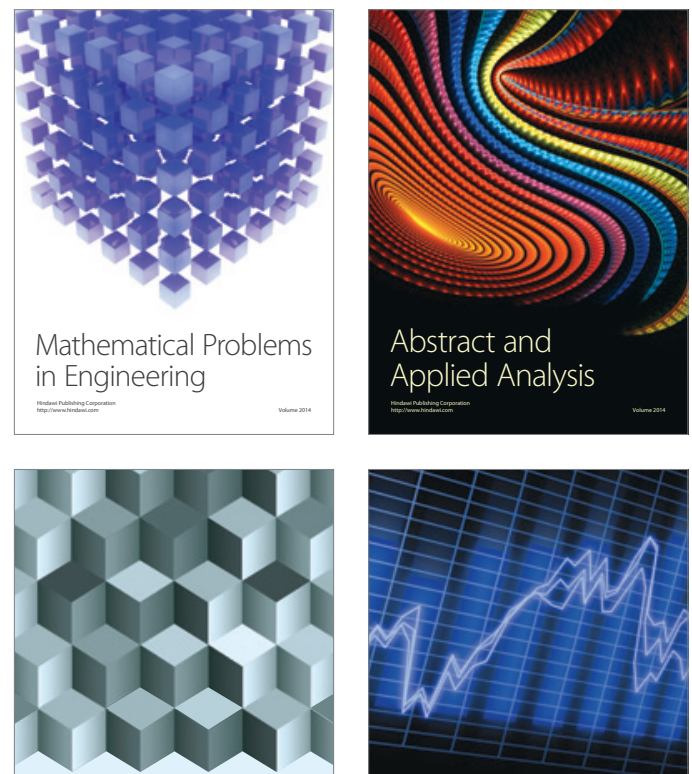

Journal of

Function Spaces

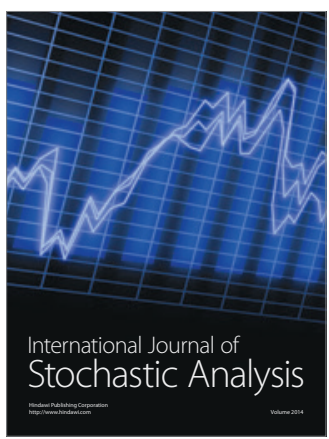

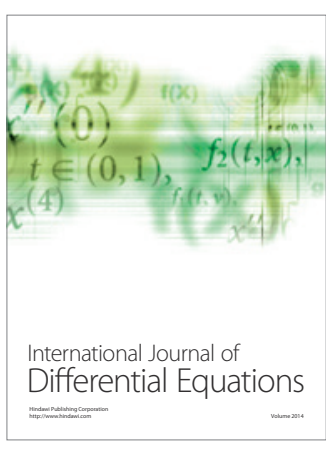
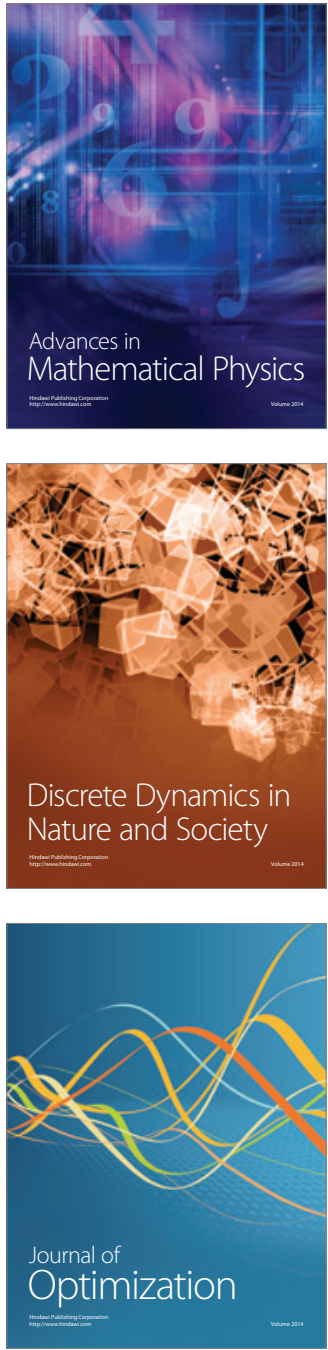